Preprint typeset in JHEP style - HYPER VERSION

ITFA-2005-17

\title{
Minisuperspace Quantization of “Bubbling AdS" and Free Fermion Droplets
}

\author{
Lars Grant ${ }^{a}$, Liat Maoz ${ }^{b}$, Joseph Marsano ${ }^{a}$, \\ Kyriakos Papadodimas ${ }^{a}$ and Vyacheslav S. Rychkov ${ }^{b}$ \\ ${ }^{a}$ Department of Physics, Harvard University \\ 17 Oxford Street, Cambridge MA 02138, USA \\ ${ }^{b}$ Institute for Theoretical Physics, University of Amsterdam \\ Valckenierstraat 65, 1018XE Amsterdam, The Netherlands \\ E-mail: Igrant@fas.harvard.edu, Imaoz@science.uva.n], \\ marsano@fas.harvard.edu, papadod@fas.harvard.edu, \\ rychkov@science.uva.n]
}

ABstract: We quantize the space of $1 / 2$ BPS configurations of Type IIB SUGRA found by Lin, Lunin and Maldacena (hep-th/0409174), directly in supergravity. We use the Crnković-Witten-Zuckerman covariant quantization method to write down the expression for the symplectic structure on this entire space of solutions. We find the symplectic form explicitly around $A d S_{5} \times S^{5}$ and obtain a $U(1)$ Kac-Moody algebra, in precise agreement with the quantization of a system of $N$ free fermions in a harmonic oscillator potential, as expected from AdS/CFT. As a cross check, we also perform the quantization around $A d S_{5} \times S^{5}$ by another method, using the known spectrum of physical perturbations around this background and find precise agreement with our previous calculation. 


\section{Contents}

1. Introduction 1

2. The LLM configurations 3

2.1 Supergravity solutions 3

2.2 Dual description in terms of fermions 5

2.3 Fermion quantization in the large $N$ limit 6

3. The $A d S$ background I - Effective action approach 10

3.1 LLM modes about $A d S_{5} \times S^{5}$

3.2 Effective action 13

4. The CWZ method of minisuperspace quantization 15

4.1 Generalities 15

4.2 Symplectic current of Type IIB SUGRA 17

5. The $A d S$ background II - CWZ approach 18

6. General droplets 19

7. Conclusions and discussion 22

A. Evaluation of the CWZ currents around $A d S_{5} \times S^{5}$

B. Evaluation of the CWZ currents around the plane wave 27

\section{Introduction}

Ever since the advent of AdS/CFT [四], particular attention has been paid to the $1 / 2$ BPS sector which, on account of the high degree of supersymmetry, is relatively simple and can often serve as a bridge connecting the gauge theory and supergravity regimes. In fact, it has been argued that this sector can be consistently decoupled [2, 3], resulting in a system that admits a description in terms of free fermions moving in a harmonic oscillator potential. This has been well understood from the gauge theory point of view, where the Lagrangian of the decoupled theory is seen to be that of a complex matrix of oscillators whose eigenvalues acquire Fermi-Dirac statistics from the integration measure. 
As a result of this, one expects a similar fermionic description of the $1 / 2$ BPS sector from the gravity point of view. How such a description might arise has been made clear in a recent paper by Lin, Lunin and Maldacena [⿴囗十), who obtained all classical 1/2 BPS solutions and demonstrated that they are naturally parametrized by planar droplets of various shapes. LLM conjectured that these droplets should be identified with phase space droplets describing semiclassical many-fermion states. In addition, [4] showed that the quantization of flux matches with the quantization of phase space area that one would expect from the fermion point of view.

We would like to take this analysis further. Is it possible to derive the full quantum structure of the fermion system directly from the LLM solutions? Such a result would be very much in line with the general spirit of AdS/CFT. ${ }^{1}$ The space of $1 / 2$ BPS solutions is simple enough that it seems plausible to address this question by a direct quantization, at least in the limit of large $N$ where an analysis within supergravity can be trusted. The usual problems associated with canonical quantization in gravity are avoided in this case because we are quantizing a space of solutions, all of which automatically satisfy the Hamiltonian constraint that is often so troubling ${ }^{2}$.

As a step in this direction, we first consider the sector of small 1/2 BPS fluctuations about the $A d S_{5} \times S^{5}$ background and construct the corresponding Hilbert space at large $N$. Performing the quantization in this case is simple since the spectrum of fluctuations is known [6] and an effective action for this sector has previously been written down [7]. Such analysis, however, is not sufficiently general to deal with fluctuations of more complicated backgrounds.

Fortunately, there exists a more general approach to quantization which does not require explicit knowledge of the spectrum. This approach takes as its starting point the symplectic form of Type IIB SUGRA, which encodes the commutation relations that must be imposed on the system. The restriction of this symplectic form to the LLM family of solutions can be computed explicitly and defines a symplectic structure that enables us to perform our quantization and study the Hilbert space about any background.

The symplectic form of supergravity can be most directly obtained by putting this theory in the canonical form, analogous to the ADM construction for pure gravity [8]. This formalism is in essence non-covariant, since it requires a particular space+time splitting of the metric, defining canonical momenta etc. In practice, it is more convenient to use an equivalent covariant method of computing the symplec-

\footnotetext{
${ }^{1}$ This problem has also been recently studied by Mandal [5], who used the D3-brane probe method to derive an action consistent with the fermionic description. In contrast, we would like to understand the correspondence between the LLM solutions and free fermion droplets directly from supergravity, i.e. without the recourse to the microscopic description of the LLM geometries in terms of D3 branes. This problem was also discussed in [5], and some suggestions concerning a possible resolution were made.

${ }^{2}$ Note also that, for this very reason, the quantization that we perform is not a moduli space quantization as we do not consider fluctuations that take us off the space of solutions in question.
} 
tic form, which was first proposed by Crnković and Witten [9] and by Zuckerman [10]. In this method one computes the symplectic form as an integral of a symplectic current, which can be derived directly from the action of the theory. In [9], explicit expressions for the symplectic currents for the Yang-Mills theory and for pure general relativity were given. It is simple to generalize these results to Type IIB supegravity. This permits us, in principle, to quantize fluctuations not only about $A d S_{5} \times S^{5}$, but also about more complicated backgrounds. After demonstrating that this procedure gives results consistent with those of the direct approach in the case of the $A d S_{5} \times S^{5}$ background, we then set up a general formalism to do precisely this.

Finally, one might worry about the validity of this method of quantization, which corresponds to a minisuperspace ${ }^{3}$ approximation of type IIB supergravity where all degrees of freedom transverse to the space of $1 / 2$ BPS configurations are artificially frozen out. However, in the case at hand we expect that our analysis indeed produces the correct Hilbert space and spectrum. That we can neglect $\alpha^{\prime}$ corrections follows because the spectrum is protected by supersymmetry and hence cannot depend on any continuous parameter ${ }^{4}$. That we can take the minisuperspace approximation within supergravity is justified because all modes transverse to the space of $1 / 2$ BPS configurations decouple in the limit of large $N$.

The outline of this paper is as follows. In section 2 , we review the $1 / 2 \mathrm{BPS}$ solutions of 4 and their parametrization by planar droplets. We also review the quantization of the relevant fermion system. In section 3, we use the effective action of [7] to quantize 1/2 BPS fluctuations about $A d S_{5} \times S^{5}$. We then review the CWZ formalism in section 4 and demonstrate its use by applying it to the same system of $1 / 2$ BPS fluctuations about $A d S_{5} \times S^{5}$ in section 5. In section 6, we initiate the program of quantizing fluctuations about backgrounds corresponding to general droplets. We then make some concluding remarks in section 7. Several calculational details have been deferred to the Appendices.

\section{The LLM configurations}

\subsection{Supergravity solutions}

In [4], all regular $1 / 2$ BPS solutions of Type IIB SUGRA with $S O(4) \times S O(4) \times \mathbb{R}$ symmetry were found. They have constant dilaton and axion and vanishing 3 -form.

\footnotetext{
${ }^{3}$ This terminology [11] has its roots in the concept of Wheeler's superspace [12], which is the space of all spatial geometries in which geometrodynamics develops. Minisuperspace quantization has been applied to models with finitely [13, 11] as well as infinitely 14 many degrees of freedom.

${ }^{4}$ One can attempt to demonstrate this more formally by noting that the $1 / 2$ BPS spectrum can be computed as the limit of an index that is currently under investigation [15].
} 
The explicit form of the solutions is ${ }^{5}$ :

$$
\begin{aligned}
d s^{2} & =-h^{-2}\left(d t+V_{i} d x^{i}\right)^{2}+h^{2}\left(d y^{2}+d x^{i} d x^{i}\right)+y e^{G} d \Omega_{3}^{2}+y e^{-G} d \tilde{\Omega}_{3}^{2}, \\
F_{5} & =F \wedge d \Omega_{3}+\tilde{F} \wedge d \tilde{\Omega}_{3}, \\
F & =d B, \quad B=B_{t}(d t+V)+\hat{B}, \\
\tilde{F} & =d \tilde{B}, \quad \tilde{B}=\tilde{B}_{t}(d t+V)+\hat{\tilde{B}},
\end{aligned}
$$

where $i=1,2, d \Omega_{3}^{2}$ and $d \tilde{\Omega}_{3}^{2}$ are the metrics on two unit 3 -spheres $S^{3}, \tilde{S}^{3}$, while $d \Omega_{3}$ and $d \tilde{\Omega}_{3}$ are the corresponding volume forms. All the unknown functions in (2.1)-(2.4) depend only on $y, x_{1}, x_{2}$ and are fixed in terms of one function $Z\left(x_{1}, x_{2}\right)$, which can only take the values $\pm \frac{1}{2}$. Namely, we have ${ }^{6}$ :

$$
\begin{aligned}
z & =\frac{1}{\pi} \frac{y^{2}}{\left(x^{2}+y^{2}\right)^{2}} * Z, \\
V_{i} & =\frac{\varepsilon_{i j}}{\pi} \frac{x_{j}}{\left(x^{2}+y^{2}\right)^{2}} * Z, \\
h^{-2} & =\frac{y}{\sqrt{1 / 4-z^{2}}}, \\
e^{2 G} & =\frac{1 / 2+z}{1 / 2-z}, \\
B_{t}=-\frac{y^{2}}{4} e^{2 G}, \quad \tilde{B}_{t}=-\frac{y^{2}}{4} e^{-2 G}, & \quad d \hat{\tilde{B}}=-\frac{y^{2}}{4} *_{3} d\left(\frac{z-1 / 2}{y^{2}}\right),
\end{aligned}
$$

where $*_{3}$ is the flat space epsilon symbol in three dimensions $y, x_{1}, x_{2}$.

The one-forms $B, \tilde{B}$ are defined up to a gauge transformation, and can be found by solving the differential equations (2.10) for $\hat{B}, \hat{\tilde{B}}$. One particular solution (arising in the gauge $\left.\hat{B}_{y}=\hat{\tilde{B}}_{y}=0\right)$ is:

$$
\begin{aligned}
& B_{i}=-\frac{y^{2}}{4\left(\frac{1}{2}-z\right)} V_{i}-\frac{\varepsilon_{i j}}{4 \pi} \frac{x_{j}}{x^{2}+y^{2}} * Z+\frac{1}{4} x_{1} \delta_{i, 2}, \\
& \tilde{B}_{i}=-\frac{y^{2}}{4\left(\frac{1}{2}+z\right)} V_{i}-\frac{\varepsilon_{i j}}{4 \pi} \frac{x_{j}}{x^{2}+y^{2}} * Z-\frac{1}{4} x_{1} \delta_{i, 2}, \\
& B_{y}=\tilde{B}_{y}=0
\end{aligned}
$$

The function $Z\left(x_{1}, x_{2}\right)$ defines 'droplets' on the $y=0$ plane. For a droplet of finite size, the spacetime asymptotically approaches $A d S_{5} \times S^{5}$ with the (common)

\footnotetext{
${ }^{5}$ We use the notation $F_{5}=\sum_{i_{1}<\ldots<i_{5}} F_{i_{1} \ldots i_{5}} d x^{i_{1}} \wedge \ldots \wedge d x^{i_{5}},\left|F_{5}\right|^{2}=\sum_{i_{1}<\ldots<i_{5}} F_{i_{1} \ldots i_{5}} F^{i_{1} \ldots i_{5}}$.

${ }^{6} \mathrm{We}$ use here the standard notation for two-dimensional convolution: $f * g(x)=\int f(x-$ $\left.x^{\prime}\right) g\left(x^{\prime}\right) d^{2} x^{\prime}$
} 
radius $R$ related to the area $A$ of the droplet by [4]

$$
A=\pi R^{4} .
$$

On the other hand, the asymptotic radius is related to the number $N$ of D3-branes making up the configuration by the standard relation (see e.g. [16])

$$
R^{4}=\frac{\kappa_{10} N}{2 \pi^{5 / 2}}
$$

This shows that the total area of the droplet must be quantized ${ }^{7}$ :

$$
\frac{A}{N}=\frac{\kappa_{10}}{2 \pi^{3 / 2}}
$$

In the situation when several droplets are present, it was shown in 四 using quantization of $F_{5}$-flux that the area of each droplet must be quantized in the same units:

$$
A_{i}=\frac{\kappa_{10}}{2 \pi^{3 / 2}} N_{i}, \quad \sum N_{i}=N
$$

In this paper we will only be considering one-droplet configurations.

\subsection{Dual description in terms of fermions}

As mentioned above, the LLM solutions corresponding to finite-size droplets are asymptotically $A d S_{5} \times S^{5}$. One can then make use of the AdS/CFT correspondence and relate these gravity solutions to $\mathcal{N}=4$ super Yang-Mills on $S^{3} \times \mathbb{R}$. As the geometries are all 1/2 BPS, the relevant operators on the Yang-Mills side are chiral primary operators with conformal weight equal to their $U(1)$ R-charge: $\Delta=J$. It was argued [2, 3] that this sector of $\mathcal{N}=4$ super Yang-Mills is actually a $U(N)$ one-matrix quantum mechanical system with a harmonic oscillator potential.

There are two equivalent ways of looking at this model. One, the 'closed string' picture, is a description of the system in terms of $N^{2}$ free harmonic oscillators with the Hamiltonian

$$
H=\frac{1}{2}\left(a^{\dagger}\right)_{j}^{i} a_{i}^{j}+\frac{1}{2} N^{2},
$$

where $\left(a^{\dagger}\right)_{j}^{i}, a_{j}^{i}$ are $N^{2}$ creation and annihilation operators, obeying the commutators:

$$
\left[\left(a^{\dagger}\right)_{j}^{i}, a_{l}^{k}\right]=\delta_{l}^{i} \delta_{j}^{k}
$$

The states

$$
\operatorname{Tr}\left[\left(a^{\dagger}\right)^{n_{1}}\right] \ldots \operatorname{Tr}\left[\left(a^{\dagger}\right)^{n_{k}}\right]|0\rangle
$$

where $k \geq 1$, and $n_{1}, n_{2}, \ldots n_{k}$ is a set of non-increasing integers between 1 and $N$, are a basis for the singlet states of the system. Every such state can also be represented in terms of a $U(N)$ Young tableaux.

\footnotetext{
${ }^{7}$ The relation $\kappa_{10}=8 \pi^{7 / 2} \ell_{P}^{4}$ is useful in comparing some of our equations to $\llbracket$.
} 
Another way of looking at this model is through the 'open string' picture. In this picture the Hamiltonian is

$$
H=\frac{1}{2} \sum_{i=1}^{N}-\partial_{\lambda_{i}}^{2}+\lambda_{i}^{2}
$$

and it describes $N$ free fermions in the harmonic oscillator potential well [3, 17]. This can also be rewritten as a system of $N$ fermions in a constant magnetic field confined to the Lowest Landau Level [3, 18]. A basis of $N$-particle wave functions is given by the Slater determinant of $N$ single particle wave functions:

$$
\psi_{\left(n_{1}, \ldots, n_{N}\right)}\left(\lambda_{1}, \ldots, \lambda_{N}\right) \sim \sum_{\sigma \in S_{N}} \operatorname{sgn}(\sigma) \prod_{i} \psi_{n_{i}}\left(\lambda_{\sigma\left(n_{i}\right)}\right) .
$$

The ground state corresponds to the fermions filling up the lowest $N$ levels. We can describe excited states by a set of $N$ non-increasing integers $n_{1}, n_{2}, \ldots n_{N}$ including zero. We move the fermion in level $N$ to $N+n_{1}$, the one in $N-1$ to $N-1+n_{2}$ etc. To this state we can associate a Young tableaux with rows of length $n_{1}, n_{2}, \ldots n_{N}$. The energy of this state is $\sum_{i} n_{i}$. Any two states described by two different sequences of integers $n_{i}$ are orthogonal. This completes the description of the Hilbert space/spectrum of this system. It is easy to write the exact partition function:

$$
Z_{\text {exact }}(\beta)=\prod_{n=1}^{N} \frac{1}{1-e^{-\beta n}}
$$

Writing this partition function in the form

$$
Z_{\text {exact }}(\beta)=\sum_{E_{i}} g\left(E_{i}\right) e^{-\beta E_{i}},
$$

we can read off the degeneracy $g\left(E_{i}\right)$ of the energy state $E_{i}$.

\subsection{Fermion quantization in the large $N$ limit}

We would like to setup a formalism to compare the quantization of the LLM solutions with the corresponding super Yang-Mills states. The correspondence is supposed to work in the large $N$ limit. On the supergravity side we will compute the symplectic form using the CWZ method, promote Poisson brackets to commutators and get a Hilbert space description. On the SYM side we will base our treatment on the "open string' description explained above. Thus we have a system of $N$ fermions in the harmonic potential, for which we already have an exact Hilbert space description. How does one pass to the large $N$ limit? In the large $N$ limit the states of the manyfermion system are well described as droplets in the one-particle phase space. It is simpler to go first one step backwards, and discuss the classical dynamics of these 
droplets $^{8}$. This discussion will lead us to the corresponding symplectic form, and thus we will be able to recover the large $N$ Hilbert space quantizing this symplectic form. The final result, Eq. (2.31), is well known; in a more general setting it is derived in 21$]$.

The harmonic potential one-fermion Hamiltonian is

$$
H=\frac{p^{2}+q^{2}}{2} \text {. }
$$

We will describe the boundary of a droplet in polar coordinates:

$$
p=r(\phi) \sin \phi, \quad q=r(\phi) \cos \phi,
$$

where we assume that $r(\phi)$ is a single-valued function. The boundary of the droplet evolves according to the one-fermion equation of motion, which in this case is simply the clockwise rotation with unit angular velocity:

$$
\dot{p}=-q, \quad \dot{q}=p .
$$

This implies that the boundary $r(\phi)$ evolves in time according to the simple equation

$$
\dot{r}=r^{\prime} \text {. }
$$

We would now like to find a symplectic form on the phase space of droplets, so that the Hamilton equation

$$
\dot{r}=\left\{r, H_{\text {tot }}\right\}
$$

would coincide with (2.26). Here $H_{t o t}$ is the total energy of the droplet state, given by the integral of the one-particle Hamiltonian:

$$
H_{\text {tot }}=\iint_{\text {droplet }} \frac{d p d q}{2 \pi \hbar} \frac{p^{2}+q^{2}}{2} .
$$

(This formula takes into account that one state occupies a phase space area of $2 \pi \hbar$ in the semiclassical description.) Notice that this $\hbar$ is the effective $1 \mathrm{~d}$ Planck constant (which has nothing to do with the $10 \mathrm{~d}$ Planck constant $\hbar_{10}$. In fact we put $\hbar_{10}=1$, as usual). This constant can be determined from

$$
A=2 \pi \hbar N
$$

where $A$ is the area of the droplet and $N$ is the total number of fermions (which is the same as the number of D3 branes in the gravity description). The total energy is easily computed in terms of $r(\phi)$ :

$$
H_{t o t}=\frac{1}{16 \pi \hbar} \int d \phi r^{4}(\phi) \equiv \frac{1}{16 \pi \hbar} \int d \phi f^{2}(\phi),
$$

\footnotetext{
${ }^{8}$ This so-called hydrodynamic approach is standard in describing edge states in the Quantum Hall Effect, see [19] and references therein. For a different approach to the large $N$ limit, using a noncommutative theory for Wigner phase space density, see [20] and references therein.
} 
where we defined $f(\phi) \equiv r^{2}(\phi)$. Let us define the following Poisson bracket (see [21])

$$
\{f(\phi), f(\tilde{\phi})\}=8 \pi \hbar \delta^{\prime}(\phi-\tilde{\phi}) .
$$

The Hamilton equation corresponding to this bracket is

$$
\dot{f}=\left\{f, H_{t o t}\right\}=f^{\prime} .
$$

This equation is equivalent to $(2.26)$, and thus we conclude that (2.31) is the correct Poisson bracket.

Although we derived this Poisson bracket for the particular one-fermion Hamiltonian (2.23), it is in fact completely general and will describe the motion of droplets of noninteracting fermions described by an arbitrary one-particle Hamiltonian $H(p, q)$. The total energy in such a general case is

$$
H_{t o t}=\int \frac{d p d q}{2 \pi \hbar} H(p, q)=\frac{1}{2 \pi \hbar} \int d \phi \int_{0}^{r(\phi)} d r r H(r \sin \phi, r \cos \phi)
$$

The evolution of the boundary of the droplet computed using the Poisson bracket (2.31) is

$$
\begin{aligned}
\dot{f} & =8 \pi \hbar \int d \tilde{\phi} \delta^{\prime}(\phi-\tilde{\phi}) \frac{\delta H_{t o t}}{\delta f(\tilde{\phi})}=8 \pi \hbar \frac{d}{d \phi}\left(\frac{\delta H_{t o t}}{\delta f(\phi)}\right) \\
& =2 \frac{d}{d \phi}[H(r(\phi) \sin \phi, r(\phi) \cos \phi)] \\
& =2\left[\left(r^{\prime} \sin \phi+r \cos \phi\right) H_{p}+\left(r^{\prime} \cos \phi-r \sin \phi\right) H_{q}\right] .
\end{aligned}
$$

We have to compare this with the evolution of the boundary which is generated by the one-fermion Hamilton equations

$$
\dot{q}=H_{p} \quad \dot{p}=-H_{q} .
$$

After a small time increment $d t$ we have

$$
\begin{aligned}
d r^{2} & =2 p d p+2 q d q=\left[2 q H_{p}-2 p H_{q}\right] d t, \\
d \phi & =r^{-2}[q d p-p d q]=-r^{-2}\left[q H_{q}+p H_{p}\right] d t \\
\left.d r^{2}\right|_{\phi=c o n s t} & =d r^{2}-\left(r^{2}\right)^{\prime} d \phi \\
& =\left[2 q H_{p}-2 p H_{q}+2\left(r^{\prime} / r\right)\left(q H_{q}+p H_{p}\right)\right] d t \\
& =2\left[\left(q+r^{\prime} \sin \phi\right) H_{p}+\left(r^{\prime} \cos \phi-p\right) H_{q}\right] d t
\end{aligned}
$$

which agrees with (2.34) as it should ${ }^{9}$.

\footnotetext{
${ }^{9}$ It should be noted that for a general Hamiltonian a region described at the initial moment of time $t=0$ by a single-valued function $r(\phi)$ can evolve at a later moment of time into a region for which $r(\phi)$ is multiple-valued. Still near $t=0$ we can describe dynamics in terms of the Poisson bracket (2.31).
} 
Now that we determined the Poisson bracket (2.31) generating classical dynamics, we can immediately write down the corresponding commutation relation multiplying by $i \hbar$. These commutation relations will have to be reproduced from the gravity side. Thus the result we should aim for is

$$
[f(\phi), f(\tilde{\phi})]=i 8 \pi \hbar^{2} \delta^{\prime}(\phi-\tilde{\phi}) \equiv i \frac{\kappa_{10}^{2}}{2 \pi^{4}} \delta^{\prime}(\phi-\tilde{\phi}) .
$$

where we expressed $\hbar$ via $\kappa_{10}$ using (2.29), (2.14).

We finish this section by showing how to construct a Hilbert space realization of (2.39). We start by expanding $f(\phi)$ in Fourier series:

$$
f(\phi)=\sum f_{n} e^{i n \phi}, \quad f_{-n}=f_{n}^{*} .
$$

The zero mode is fixed in terms of the droplet area:

$$
f_{0}=\frac{A}{\pi} .
$$

The $n \neq 0$ modes correspond to the area-preserving deformations. Substituting (2.40) in (2.39), we get commutation relation between the corresponding Fourier coefficients:

$$
\left[f_{n}, f_{m}\right]=-\frac{\kappa_{10}^{2}}{4 \pi^{5}} n \delta_{n+m}
$$

This is just a $U(1)$ Kac-Moody algebra ${ }^{10}$. The Hilbert space of the theory can be constructed as a bosonic Fock space with annihilation and creation operators $c_{n}, c_{n}^{\dagger}$ where

$$
c_{n}=\left(\frac{\kappa_{10}^{2}}{4 \pi^{5}} n\right)^{-1 / 2} f_{-n}, \quad n>0
$$

Of course there is no contradiction with the fermionic statistics of the individual particles: the droplet exitations are collective modes and are free to satisfy any statistics.

Finally, as a check of the validity of this semiclassical description we can compute the partition function of many fermions in a harmonic potential using the semiclassical method and compare with the result of the exact quantization. Using the bosonic Hilbert space constructed above by the operators $c_{n}, c_{n}^{\dagger}$ we find:

$$
Z_{\text {semiclassical }}(\beta)=\prod_{n=1}^{\infty} \frac{1}{1-e^{-\beta n}} .
$$

\footnotetext{
${ }^{10}$ One can also add to the $f_{n}$ 's an energy-momentum tensor using the Sugawara construction. It turns out to be proportional to (2.30). Thus $f(\phi)$ is related to a free chiral boson $X(\phi)$ through $f(\phi) \sim \partial_{\phi} X(\phi)$, as can also be seen from (2.39). In the context of the Quantum Hall Effect, this anomaly equation expresses the non-conservation of the charge density on the boundary in presence of an external electric field [22].
} 
Comparing with (2.21) we see that the semiclassical method reproduces the correct spectrum of the theory $E_{i}=1,2,3, \ldots$ and the exact degeneracies of these states provided that $E<N$. For higher energies the degeneracies predicted by the semiclassical quantization do not agree with those of the exact quantization. But since our calculations in gravity are valid only for large $N$, we will always be in the regime where the semiclassical approximation is reliable.

\section{The $A d S$ background I - Effective action approach}

In this section, we consider small $1 / 2$ BPS fluctuations about the $A d S_{5} \times S^{5}$ background and quantize them directly via an effective action obtained from Type IIB SUGRA. This is not too difficult to do as the spectrum of modes on $A d S_{5} \times S^{5}$ is well known [6] and, moreover, the effective action for the 1/2 BPS fluctuations has been worked out to the order in which we are interested by [7]. Thus, to compare with the fermion quantization of the previous section, we need only to relate the LLM parametrization of $1 / 2$ BPS fluctuations in terms of deformations of a droplet to the modes of [6], which differ by a diffeomorphism. We review the LLM description of $A d S_{5} \times S^{5}$ and $1 / 2$ BPS fluctuations about this background and connect to the modes of [6] in the first subsection. We then utilize the effective action of [7] to perform the quantization. The result of this quantization, Eq. (3.26), perfectly agrees with what we expect from the fermion side, Eq. (2.42).

\subsection{LLM modes about $A d S_{5} \times S^{5}$}

In the language of LLM, the $A d S_{5} \times S^{5}$ background corresponds to a circular droplet in the $\left(x_{1}, x_{2}\right)$ plane with radius $r_{0}=R_{A d S}^{2}$ that we set to 1 by convention. Evaluating the various functions appearing in the metric for such a droplet and using polar coordinates $r, \phi$ on the $\left(x_{1}, x_{2}\right)$ plane, we find

$$
\begin{aligned}
z(r, y) & =\frac{r^{2}-1+y^{2}}{2 \sqrt{\left(r^{2}+1+y^{2}\right)-4 r^{2}}}, \\
V_{r} & =0 \\
V_{\phi} & =\frac{1}{2}\left(\frac{r^{2}+1+y^{2}}{\sqrt{\left(r^{2}+1+y^{2}\right)^{2}-4 r^{2}}}-1\right) .
\end{aligned}
$$

If we make the change of coordinates

$$
\begin{aligned}
& y=\sinh \rho \sin \theta, \\
& r=\cosh \rho \cos \theta, \\
& \phi=\tilde{\phi}+t .
\end{aligned}
$$

then the LLM metric (2.1) becomes that of $A d S_{5} \times S^{5}$ in global coordinates

$$
d s^{2}=-\cosh ^{2} \rho d t^{2}+d \rho^{2}+\sinh ^{2} \rho d \Omega_{3}^{2}+d \theta^{2}+\cos ^{2} \theta d \tilde{\phi}^{2}+\sin ^{2} \theta d \tilde{\Omega}_{3}^{2},
$$


and the five-form becomes

$$
F_{5}=\cosh \rho \sinh ^{3} \rho d t \wedge d \rho \wedge d \Omega_{3}+\cos \theta \sin ^{3} \theta d \theta \wedge d \tilde{\phi} \wedge d \tilde{\Omega}_{3} .
$$

We now consider perturbations of this background corresponding to small ripples of the droplet. The boundary of the perturbed droplet in polar coordinates is given by $r(\phi)=1+\delta r(\phi)$. We expand $\delta r(\phi)$ in Fourier series ${ }^{11}$ :

$$
\delta r(\phi)=\sum_{n \neq 0} a_{n} e^{i n \phi}, \quad a_{n}^{*}=a_{-n}
$$

Using (2.5), (2.6), it is easy to find the first order shifts in the functions $z, V_{i}$ due to the presence of these ripples:

$$
\begin{aligned}
\delta z(r, \phi, y) & =-\sum_{n} \frac{2 y^{2} a_{n} e^{i n \phi}}{B^{2}}\left(\frac{1+|n| \sqrt{1-A^{2}}}{\left(1-A^{2}\right)^{3 / 2}}\right)\left[\frac{1-\sqrt{1-A^{2}}}{A}\right]^{|n|}, \\
\delta V_{r}(r, \phi, y) & =\sum_{n} \frac{2 i n a_{n} e^{i n \phi}}{B^{2} A \sqrt{1-A^{2}}}\left[\frac{1-\sqrt{1-A^{2}}}{A}\right]^{|n|}, \\
\delta V_{\phi}(r, \phi, y) & =\sum_{n} \frac{2 r a_{n} e^{i n \phi}}{B^{2}\left(1-A^{2}\right)^{3 / 2}}\left[(r-A)+\left(r-A^{-1}\right)|n| \sqrt{1-A^{2}}\right]\left[\frac{1-\sqrt{1-A^{2}}}{A}\right]^{|n|},
\end{aligned}
$$

where

$$
\begin{aligned}
& A \equiv \frac{2 r}{r^{2}+y^{2}+1} \\
& B \equiv r^{2}+y^{2}+1
\end{aligned}
$$

Inserting these variations into (2.1), we obtain the metric perturbations which correspond to the excitation of ripples on the droplet in the fermi picture. To identify these perturbations with the modes of Kim et al. [6], two additional operations are required. First of all, we have to move to global $A d S$ coordinates via (3.2). In this way we arrive at a certain metric perturbation $h_{m n}$, which is still not in the Kim et al. form. To achieve total coincidence, we have to perform an additional linear gauge transformation

$$
h_{m n} \rightarrow h_{m n}-\left(\nabla_{m} \xi_{n}+\nabla_{n} \xi_{m}\right)
$$

\footnotetext{
${ }^{11}$ The zero mode is absent, since the deformation should be area preserving (to the first order in $\delta r)$.
} 
for the particular choice of $\xi_{i}$ with nonzero components

$$
\begin{aligned}
& \xi_{t}=-\sum_{n} \frac{i n}{|n|} a_{n} e^{i n(\tilde{\phi}+t)}\left(\frac{\cos \theta}{\cosh \rho}\right)^{|n|}, \\
& \xi_{\rho}=-\sum_{n} \frac{2 \cos ^{2} \theta \tanh \rho}{\cosh (2 \rho)-\cos (2 \theta)} a_{n} e^{i n(\tilde{\phi}+t)}\left(\frac{\cos \theta}{\cosh \rho}\right)^{|n|}, \\
& \xi_{\theta}=\sum_{n} \frac{\sin (2 \theta)}{\cosh (2 \rho)-\cos (2 \theta)} a_{n} e^{i n(\tilde{\phi}+t)}\left(\frac{\cos \theta}{\cosh \rho}\right)^{|n|} .
\end{aligned}
$$

The resulting metric perturbations then take the form

$$
h_{\mu \nu}=\sum_{n \neq 0}\left(-\frac{6}{5}|n| s_{n} Y_{n} g_{\mu \nu}+\frac{4}{|n|+1} Y_{n} \nabla_{(\mu} \nabla_{\nu)} s_{n}\right), \quad h_{\alpha \beta}=\sum_{n \neq 0} 2|n| s_{n} Y_{n} g_{\alpha \beta}
$$

where $\mu, \nu$ run over the $A d S_{5}$ directions, $\alpha, \beta$ run over the $S^{5}$ directions, $g_{m n}$ is the unperturbed $A d S_{5} \times S^{5}$ metric, the covariant derivatives are computed with respect to the metric $g_{m n}$, and

$$
\begin{aligned}
s_{n} & =\frac{|n|+1}{2|n| \cosh ^{|n|} \rho} a_{n} e^{i n t}, \\
Y_{n} & =e^{i n \tilde{\phi}} \cos ^{|n|} \theta .
\end{aligned}
$$

Written in this form, the metric perturbations can be identified with a subclass of modes studied in [6], [7]. Thus, the modes of [6], [7] have the same functional dependence as (3.10) with functions $s_{n}, Y_{n}$ satisfying the differential equations

$$
\nabla_{S^{5}}^{2} Y_{n}=-n(n+4) Y_{n}, \quad \nabla_{A d S_{5}}^{2} s_{n}=n(n-4) s_{n}
$$

so that, for instance, the $Y_{n}$ are $S^{5}$ spherical harmonics. Our $s_{n}$ and $Y_{n}$ correspond, not surprisingly, to the subclass of solutions of these equations that are constant on $S^{3}$ and $\tilde{S}^{3}$.

The perturbation of the 4-form potential could be in principle analyzed using the same method (expressing perturbations in LLM coordinates, passing to the global coordinates, and following up by a gauge transformation). However, this is unnecessary, because the end result of this computation can be uniquely reconstructed from the knowledge of metric perturbations (3.10). Namely, the 4-form potential perturbations can be written in the gauge $\nabla^{\alpha} a_{\alpha m n p}=0$ as [6]

$$
\begin{aligned}
& \delta a_{\alpha \beta \gamma \delta}=-\epsilon_{\alpha^{\prime} \alpha \beta \gamma \delta} s_{n} \nabla^{\alpha^{\prime}} Y_{n} \\
& \delta a_{\mu \nu \rho \lambda}=\epsilon_{\mu^{\prime} \mu \nu \rho \lambda} Y_{n} \nabla^{\mu^{\prime}} s_{n}
\end{aligned}
$$

where $\epsilon_{\alpha^{\prime} \alpha \beta \gamma \delta}\left(\epsilon_{\mu^{\prime} \mu \nu \rho \lambda}\right)$ is the curved-space $\epsilon$ symbol on $S^{5}\left(A d S_{5}\right)$. 


\subsection{Effective action}

The effective second-order action describing perturbations of $A d S_{5} \times S^{5}$ of the form (3.10), (3.13) has been derived in [7] and has the form

$$
S=\frac{1}{2 \kappa_{10}^{2}} \sum_{I} \int d^{5} x \sqrt{-g_{A d S}} \frac{A_{I}}{2}\left[-\left(\nabla s_{I}\right)^{2}-n(n-4)\left(s_{I}\right)^{2}\right],
$$

where the index $I$ labels spherical harmonics. This effective action was only derived in [7] for modes with $n \geq 2$, where $n=n(I)$ is the parameter in the eigenvalue equations (3.12). As a result, we leave the $n=1$ mode out of consideration in this section. It will however be covered by the alternative treatment in Sec. 5 .

The action (3.14) was derived under the assumption that the spherical harmonics are real and satisfy an orthogonality relation

$$
\int_{S^{5}} Y_{I} Y_{I^{\prime}}=z_{I} \delta_{I, I^{\prime}}
$$

The action is obtained by expanding the Type IIB SUGRA action to the second order in perturbations and performing the $S^{5}$ integration $^{12}$. The constants $A_{I}$ are given by:

$$
A_{I}=32 \frac{n(n-1)(n+2)}{n+1} z_{I} .
$$

In this subsection, we will apply this effective action to quantize the LLM family of $1 / 2$ BPS fluctuations. A minor complication arises since angular momentum conservation prevents a direct restriction to the sector in question at the level of the action so we will have to include modes that rotate in the opposite direction. After quantization, though, it will be easy to make the appropriate truncation of the quantized Hilbert space.

We now proceed using the real modes

$$
\begin{array}{ll}
s_{n, 1}=\frac{n+1}{2 n \cosh ^{n} \rho} x_{n}(t), & Y_{n, 1}=\cos ^{n} \theta \cos (n \phi), \\
s_{n, 2}=\frac{(n+1)}{2 n \cosh ^{n} \rho} y_{n}(t), & Y_{n, 2}=\cos ^{n} \theta \sin (n \phi) .
\end{array}
$$

These modes are chosen because they are related to (3.11):

$$
s_{n} Y_{n}+s_{-n} Y_{-n}=\sum_{i=1}^{2} s_{n, i} Y_{n, i}
$$

for the particular choice of real functions $x_{n}(t)$ and $y_{n}(t)$

$$
x_{n}(t)=a_{n} e^{i n t}+\text { c.c. }, \quad y_{n}(t)=i a_{n} e^{i n t}+\text { c.c. }
$$

\footnotetext{
${ }^{12}$ More precisely, the expansion is done in the 'actual' IIB SUGRA action [23] in which the selfduality constraint on the 5 -form is enforced via an auxiliary field.
} 
Substituting (3.17) into the effective action (3.14) and performing the spatial integrations, we obtain the following effective action for $x_{n}(t), y_{n}(t)$ :

$$
S=\frac{2 \pi^{5}}{\kappa_{10}^{2}} \sum_{n \geq 2} \int d t\left[\frac{1}{n^{2}}\left(\dot{x}_{n}^{2}+\dot{y}_{n}^{2}\right)-\left(x_{n}^{2}+y_{n}^{2}\right)\right] \text {. }
$$

The quantization of this system is now straightforward. We have the following equaltime canonical commutators:

$$
\left[x_{n}, \dot{x}_{m}\right]=\left[y_{n}, \dot{y}_{m}\right]=i n^{2}\left(\frac{\kappa_{10}^{2}}{4 \pi^{5}}\right) \delta_{n, m} .
$$

Representing $x_{n}(t), y_{n}(t)$ as

$$
x_{n}=c_{n} e^{i n t}+h . c ., \quad y_{n}=d_{n} e^{i n t}+h . c .,
$$

we get the following nonzero commutation relations for $c_{n}, d_{n}$

$$
\left[c_{n}^{\dagger}, c_{m}\right]=\left[d_{n}^{\dagger}, d_{m}\right]=\frac{\kappa_{10}^{2}}{8 \pi^{5}} n \delta_{n, m}
$$

As discussed before, the full Hilbert space of the system (3.20) contains both the 1/2BPS fluctuations of interest as well as those carrying opposite angular momentum. We thus have to truncate this Hilbert space according to (3.19). In order to do this consistently, we put

$$
c_{n}=a_{n}+b_{n}, \quad d_{n}=i\left(a_{n}-b_{n}\right) .
$$

Then from (3.23) we get the following commutators for $a_{n}, b_{n}$ :

$$
\left[a_{n}^{\dagger}, a_{m}\right]=\left[b_{n}^{\dagger}, b_{m}\right]=\frac{\kappa_{10}^{2}}{16 \pi^{5}} n \delta_{n, m} .
$$

Since all commutators between $a_{n}$ and $b_{m}$ vanish, the $b_{n}$ sector decouples and it is consistent to truncate the full Hilbert space keeping only the states in the $a_{n}$ sector. In the classical theory this corresponds to setting $b_{n}=0$, which is consistent with (3.19). The commutation relation for $a_{n}$ in (3.25), which can be rewritten as

$$
\left[a_{n}, a_{m}\right]=-\frac{\kappa_{10}^{2}}{16 \pi^{5}} n \delta_{n+m}
$$

is easily seen to be consistent with (2.42) using the fact that $\delta\left(r^{2}\right)=2 \delta r$, and thus $f_{n}=2 a_{n}$.

Finally, we note that the Hamiltonian density for the system (3.20), (3.19) is also easy to compute:

$$
H=\frac{16 \pi^{5}}{\kappa_{10}^{2}} \sum_{n \geq 2} a_{n} a_{-n}
$$

and agrees with what one obtains from LLM (2.32). 


\section{The CWZ method of minisuperspace quantization}

The quantization method described in the previous section does not seem to be applicable for the general droplet case. As motivated in the introduction, in this section we will review and apply to Type IIB SUGRA the covariant phase space approach of Crnković-Witten-Zuckerman. It is this method that will be used in the rest of the paper.

\subsection{Generalities}

Crnković and Witten [9] and Zuckerman [10] (see also [24]) proposed an alternative approach to quantizing Lagrangian theories which is equivalent to the usual Hamiltonian formalism and is particularly suited for minisuperspace quantization. The starting point of the CWZ approach is that, in a general field theory, the canonical phase space is in one-to-one correspondence, and thus can be identified, with the space of solutions of the classical field equations. Thus we should be able to define the symplectic form needed for quantization directly on the space of solutions, without recourse to some specific canonical variables.

In this approach, the symplectic form is computed as an integral of a specific symplectic current $J^{l}$ over an initial hypersurface $\Sigma$ :

$$
\omega=\int d \Sigma_{l} J^{l} .
$$

The simplest example is that of the free scalar field $\phi$ in Minkowski space, in which the symplectic current is given by

$$
J^{l}(x)=\partial^{l} \delta \phi(x) \wedge \delta \phi(x) .
$$

Here, using the notation of [9], $\delta \phi$ is an element of the tangent space to the space of solutions. In particular, $\delta \phi$ satisfies the linearized equations of motion (which in this simplest linear situation coincide with the original ones). By evaluating $\delta \phi$ at a point $x$, we get a one-form on the phase space, $\delta \phi(x)$. Analogously $\partial^{l} \delta \phi(x)$ is also a one-form for each $x$. More forms can be constructed using the exterior product $\wedge$ and the exterior differentiation $\delta$. We see that $\omega$ defined by (4.1) is a two-form on the phase space. Moreover, it is closed because $\delta(\delta \phi(x))=0$. It is also invariant under variations of $\Sigma$, because the $J^{l}$ as defined by (4.2) is conserved: $\partial_{l} J^{l}=0$. Note that one needs the equations of motion to show this and hence it is true only when the symplectic form is evaluated on the phase space of solutions.

To complete the free scalar quantization, one should choose a particular parametrization of the solutions to the Klein-Gordon equation, e.g. by expanding solutions into plane waves, and compute the symplectic form (4.1) in terms of the coefficients of such an expansion. The Poisson brackets following from the computed 
symplectic form are then promoted to commutators using the Dirac prescription. The resulting quantization is completely equivalent to the usual Hamiltonian approach.

It turns out that the above method is not limited to free scalar fields, but can be adapted to a wide range of theories, including those with gauge symmetries, where the symplectic form has to satisfy an additional constraint of gauge invariance. In [9], appropriate symplectic currents were written down for Abelian and non-Abelian gauge theories as well as for pure gravity; for recent applications of the method to other physical theories see [25]. For theories with several fields it becomes easier to compute the symplectic current using the following general method [10], [26]. We will assume that the Lagrangian $L=L\left(\phi_{A}, \partial_{l} \phi_{A}\right)$ (where the index $A$ numbers the fields) does not contain second- and higher-order derivatives, so that the classical equations of motion are

$$
\frac{\partial L}{\partial \phi_{A}}-\partial_{l} \frac{\partial L}{\partial \partial_{l} \phi_{A}}=0
$$

Under these conditions, the symplectic current is defined by ${ }^{13}$

$$
J^{l}=\delta\left[\frac{\partial L}{\partial \partial_{l} \phi_{A}}\right] \delta \phi_{A}=\delta\left[\frac{\partial L}{\partial \partial_{l} \phi_{A}} \delta \phi_{A}\right] .
$$

(From now on we, following [9], will usually omit the wedge product sign, treating $\delta \phi$ 's as anticommuting objects. Of course, wedge products can be reinstated at any moment, if desired.) It is obvious from the second representation that $J^{l}$ is closed: $\delta J^{l}=0$. It is also conserved, due to the equations of motion:

$$
\begin{aligned}
\partial_{l} J^{l} & =\delta\left[\partial_{l} \frac{\partial L}{\partial \partial_{l} \phi_{A}} \delta \phi_{A}+\frac{\partial L}{\partial \partial_{l} \phi_{A}} \delta \partial_{l} \phi_{A}\right] \\
& =\delta\left[\frac{\partial L}{\partial \phi_{A}} \delta \phi_{A}+\frac{\partial L}{\partial \partial_{l} \phi_{A}} \delta \partial_{l} \phi_{A}\right]=\delta[\delta L]=0 .
\end{aligned}
$$

The symplectic form is defined via the symplectic current by the same Eq. (4.1) as before. This form is closed because $J^{l}$ is closed, and it is $\Sigma$-independent because $J^{l}$ is conserved ${ }^{14}$. It is slightly more complicated to show that in theories with gauge symmetries the symplectic form so defined will be gauge invariant. For specific equations it was shown in [9], and a general argument can be found in [10, [26].

\footnotetext{
${ }^{13}$ Actually, what we define is a vector density and hence is appropriate to be integrated without the usual $\sqrt{-g}$ factor.

${ }^{14} \mathrm{~A}$ subtlety arises if the theory in question is defined on a manifold with spatial infinity. Strictly speaking, the conservation of $J^{l}$ only implies that $\omega$ is invariant under variations which keep a part of $\Sigma$ near infinity fixed. A related problem is that the symplectic current as defined by (4.4) can lead to a divergent symplectic form. In this case a boundary term should be added to (4.1) to compensate the divergence. It turns out, however, that in all cases analysed in this paper the symplectic form defined by the most natural expressions (4.1), (4.4) is manifestly finite. We take it as an indication that in our case no boundary terms are necessary, and that the symplectic form is invariant also under shifts of $\Sigma$ at infinity. However, since our plane wave computation in Sec. 6 does not match the expected answer, it is possible that the situation is not so simple.
} 
The sign and normalization of the definitions (4.1), (4.4) can be checked by applying these formulas to the one-particle classical mechanics, in which case they give, correctly

$$
L=\int d t\left(\frac{1}{2} \dot{q}^{2}-V(q)\right), \quad \omega=J^{t}=\delta \dot{q} \wedge \delta q=\delta p \wedge \delta q
$$

\subsection{Symplectic current of Type IIB SUGRA}

The LLM solutions satisfy equations of motion that can be derived from the following action:

$$
S=\frac{1}{2 \kappa_{10}^{2}} \int d^{10} x \sqrt{-g}\left(R-4\left|F_{5}\right|^{2}\right),
$$

We can proceed using this action if we impose the selfduality constraint $F_{5}=* F_{5}$ on the solutions. The presence of this constraint does not modify the underlying symplectic form of the theory, which can be computed from the action (4.7) $)^{15}$.

Using the general formulas of the previous subsection, the symplectic form will be equal to

$$
\omega=\frac{1}{2 \kappa_{10}^{2}} \int d \Sigma_{l}\left(J_{G}^{l}+J_{F}^{l}\right)
$$

where $J_{G}^{l}$ and $J_{F}^{l}$ are symplectic currents constructed from the gravity and 5-form parts of the Langrangian using Eq. (4.4).

To find the gravity current, we need first to remove the second derivatives from the Einstein-Hilbert action, which is equivalent to adding the Gibbons-Hawking boundary term [27. The resulting first-derivative action can be conveniently written in the so-called $\Gamma \Gamma-\Gamma \Gamma$ form (see e.g. [28]):

$$
L=\sqrt{-g} g^{i k}\left[\Gamma_{i l}^{m} \Gamma_{k m}^{l}-\Gamma_{i k}^{l} \Gamma_{l m}^{m}\right] .
$$

(The sign is correct provided that the signature is mostly + .) We take the inverse metric components $g^{m n}$ as our basic fields. Varying (4.9) with respect to $\partial_{l} g^{m n}$, it is easy to compute:

$$
\begin{aligned}
\frac{\partial L}{\partial \partial_{l} g^{m n}} & =\sqrt{-g}\left[-\Gamma_{m n}^{l}+\delta_{(m}^{l} \Gamma_{n) k}^{k}+\frac{1}{2} g_{m n}\left(g^{i k} \Gamma_{i k}^{l}-g^{l i} \Gamma_{i k}^{k}\right)\right], \\
\frac{\partial L}{\partial \partial_{l} g^{m n}} \delta g^{m n} & =-\Gamma_{m n}^{l} \delta\left[\sqrt{-g} g^{m n}\right]+\Gamma_{m n}^{n} \delta\left[\sqrt{-g} g^{l m}\right] .
\end{aligned}
$$

Thus we get

$$
J_{G}^{l}=-\delta \Gamma_{m n}^{l} \wedge \delta\left[\sqrt{-g} g^{m n}\right]+\delta \Gamma_{m n}^{n} \wedge \delta\left[\sqrt{-g} g^{l m}\right],
$$

\footnotetext{
${ }^{15}$ In particular, one can perform the following analysis with the 'actual' IIB action [23] in which the selfduality constraint is imposed via an auxiliary field.
} 
which is the old result of Crnković and Witten [9 $]^{16}{ }^{17}$. Notice that [9] defines the symplectic form as $\int d \Sigma_{l} \sqrt{-g} J^{l}$, so that our symplectic currents differ from [9] by a factor of $\sqrt{-g}$.

To find the 5-form current, we take the potentials $A_{\left|k_{1} \ldots k_{4}\right|}\left(F_{5}=d A\right)$ as our basic fields ${ }^{18}$. Applying (4.4), we get immediately

$$
J_{F}^{l}=-8 \delta\left(\sqrt{-g} F^{l\left|k_{1} \ldots k_{4}\right|}\right) \delta A_{\left|k_{1} \ldots k_{4}\right|} .
$$

Now that we computed the symplectic current, we can simplify it using the selfduality constraint, which can be written as

$$
F^{l_{1} \ldots l_{5}}=\frac{1}{\sqrt{-g}} \varepsilon^{l_{1} \ldots l_{5}\left|m_{1} \ldots m_{5}\right|} F_{\left|m_{1} \ldots m_{5}\right|},
$$

where $\varepsilon^{\cdots}$ is the flat 10 -dimensional epsilon symbol. Thus we have

$$
J_{F}^{l}=-8 \varepsilon^{l\left|k_{1} \ldots k_{4}\right|\left|m_{1} \ldots m_{5}\right|} \delta F_{\left|m_{1} \ldots m_{5}\right|} \delta A_{\left|k_{1} \ldots k_{4}\right|} .
$$

\section{The $A d S$ background II - CWZ approach}

We will now quantize small $1 / 2$ BPS fluctuations about $A d S_{5} \times S^{5}$ using the CWZ method described in the previous section. Thus we have to compute the symplectic currents (4.11), (4.12) and integrate them to get the symplectic form (4.8). The most direct approach would be to use the LLM parametrization of the solutions in terms of the functions $h, V_{i}$ etc. This approach is pursued in the next section. In this section, we will use a hybrid approach. Namely, we will once again use the modes of Kim et al. [6] which were related to the LLM droplet deformations (3.5) in Sec. 3, Eqs. (3.10) and (3.13). However, we will not rely on the effective action to perform the quantization, as we did in Sec. 3. Instead, we will use the Kim et al. modes to evaluate the CWZ symplectic currents and the symplectic form, which we then quantize.

In order to utilize the $t$-independence of the LLM solutions, we will make the most natural choice of the hypersurface $\Sigma=\{t=$ const $\}$ in (4.8). Thus we only need to calculate $J^{t}$ to derive the symplectic form $\omega$. The details of this calculation can be found in Appendix A. The contribution from the gravity and 5-form current

\footnotetext{
${ }^{16}$ Actually, our result differs from that of $[9]$ by an overall sign, which can be traced to a different convention for the metric signature.

${ }^{17} \mathrm{It}$ is stated in [26] that this result is also contained, in a disguised form, in [29], but we were not able to locate it in that paper.

${ }^{18}$ The notation $\left|i_{1} \ldots i_{n}\right|$ means that the indices have to be ordered: $i_{1}<\ldots<i_{n}$. Thus we have $A=A_{\left|i_{1} \ldots i_{4}\right|} d x^{i_{1}} \wedge \ldots \wedge d x^{i_{4}}=\frac{1}{4 !} A_{i_{1} \ldots i_{4}} d x^{i_{1}} \wedge \ldots \wedge d x^{i_{4}}$. The same ordering is assumed, e.g., in the summation in Eq. (4.12).
} 
turn out to be, respectively

$$
\begin{aligned}
& \int_{t=\mathrm{const}} J_{G}^{t}=8 \pi^{5} i \sum_{n \neq 0} \frac{n^{2}-3|n|-8}{(|n|-1)(|n|+2) n} a_{n} \wedge a_{-n}, \\
& \int_{t=\mathrm{const}} J_{F}^{t}=8 \pi^{5} i \sum_{n \neq 0} \frac{n^{2}+5|n|+4}{(|n|-1)(|n|+2) n} a_{n} \wedge a_{-n} .
\end{aligned}
$$

Notice that the coefficients $a_{n}$ of the Fourier series (3.5) are treated here as one-forms on the LLM phase space. Substituting these into (4.8), we obtain the symplectic form

$$
\omega=\frac{8 \pi^{5} i}{\kappa_{10}^{2}} \sum_{n \neq 0} \frac{1}{n} a_{n} \wedge a_{-n} .
$$

Inverting this symplectic form, we get the nonzero Poisson brackets:

$$
\left\{a_{m}, a_{n}\right\}=-\frac{\kappa_{10}^{2}}{16 \pi^{5}} i n \delta_{m+n} .
$$

The commutators obtained from this bracket by the Dirac prescription $[]=,i\{$, agree with the result (3.26) previously obtained by the effective action method, and thus also with what we expect from the fermion side, Eq. (2.42).

It should be noted that for $n=1$ Eqs. (5.1) and (5.2) are formally divergent. This divergence is due to the insufficiently fast individual decay of $J_{G}^{t}$ and $J_{F}^{t}$ at large $\rho$ (see Eqs. (A.7), (A.9), (A.14) in Appendix A). It is easy to check, however, that in the combined integral $\int J_{G}^{t}+J_{F}^{t}$ the divergence cancels, and the finite result of this integration agrees with Eq. (5.3), which is thus true also for $n=1$.

\section{General droplets}

In this section we will apply the CWZ method to write down the symplectic form for the family of LLM configurations around an arbitrary droplet. In this case, unlike for the $A d S_{5} \times S^{5}$ case we analyzed in Sec. 5, we don't have at our disposal a natural basis of linear perturbations around this solution (analogous to the spherical harmonic basis of Kim et al. [6] around $A d S_{5} \times S^{5}$ ). In order to have general expressions, we will express the symplectic current in terms of the LLM ansatz functions $h, V_{i}$ etc. As a concrete example, we then apply these general expressions to the plane wave background.

Let us start from the gravitational piece of the symplectic current. Just like in Sec. 5, we will use $\Sigma=\{t=$ const $\}$ in (4.8). Thus we only need to calculate $J^{t}$. Taking the general expression (4.11) and plugging in the expressions for the variations of the metric and Christoffel symbols, we find the following expression:

$$
J_{G}^{t}=y^{3}\left[-\frac{1}{4} \delta\left(V_{i} \partial_{i} h^{-4}\right) \delta\left(h^{4}\right)+3 \delta\left(V_{i} G_{, i}\right) \delta G+\delta\left(h^{-4} W_{i j} V_{j}\right) \delta V_{i}-4 \delta\left(\partial_{i} \ln h\right) \delta V_{i}\right]
$$


(where $i, j=1,2, W_{i j} \equiv \partial_{i} V_{j}-\partial_{j} V_{i}$ ). The last term integrates to zero because $\partial_{i} V_{i}=0$, and can be dropped. The first two terms add up nicely if one expresses $h$ and $G$ via $z$ from (2.7), (2.8). Remembering that $(\delta z)^{2}=0$, we get:

$$
J_{G}^{t}=y^{3}\left[\frac{3 / 4+z^{2}}{\left(1 / 4-z^{2}\right)^{2}} \delta\left(V_{i} z_{, i}\right) \delta z+\delta\left(h^{-4} W_{i j} V_{j}\right) \delta V_{i}\right] .
$$

Similarly, using the expressions in (4.14) and the form of the $F_{5}$ ansatz (2.2)(2.4), the $F_{5}$ current can be simplified as follows:

$$
J_{F}^{t}=4 \varepsilon^{a b c}\left(\delta B_{a} \delta \tilde{F}_{b c}-\delta \tilde{B}_{a} \delta F_{b c}\right) .
$$

where $\varepsilon^{a b c}$ is the flat space epsilon symbol in three dimensions $y, x_{1}, x_{2}$. Since we will be working in the $B_{y}=\tilde{B}_{y}=0$ gauge, this expression can be simplified even further:

$$
J_{F}^{t}=8 \varepsilon^{i j}\left(\partial_{y} \delta B_{i} \delta \tilde{B}_{j}-\delta B_{i} \partial_{y} \delta \tilde{B}_{j}\right) .
$$

Since the derived symplectic currents do not depend on the $S^{3}, \tilde{S}^{3}$ variables, the symplectic form (4.8) can be simplified to

$$
\omega=\frac{\left(2 \pi^{2}\right)^{2}}{2 \kappa_{10}^{2}} \int d x_{1} d x_{2} d y\left(J_{G}^{t}+J_{F}^{t}\right) .
$$

Eqs. (6.2), (6.4), (6.5) implicitly define the symplectic form on the whole LLM class of solutions. We would like to make an important remark here, namely that the above expressions in Eqs. (6.2), (6.4) are the symplectic currents derived for the IIB SUGRA action (4.7) in the 10-dimensional bulk, with second derivatives removed from the Einstein-Hilbert term. It is possible, as we noted in Sec. 因, that this action has to be supplemented by extra boundary terms, which would give an extra contribution to the symplectic currents. For a discussion of this issue, in a slightly different context, see [30].

Explicit evaluation of the expression (6.5) in the general droplet case is not easy, and is postponed to the future. Below we demonstrate how to apply these expressions to evaluate the symplectic form around the plane wave ${ }^{19}$.

The plane wave background corresponds to a droplet which covers the entire lower half-plane, i.e. $Z\left(x_{1}, x_{2}\right)=\frac{1}{2} \operatorname{sign} x_{2}$. The various functions specifying the solution are given by:

$$
\begin{array}{rlrl}
z & =\frac{x_{2}}{2 r}, & r \equiv \sqrt{x_{2}^{2}+y^{2}}, \\
V_{1} & =-\frac{1}{2 r}, & & V_{2}=0, \\
h^{-2} & =2 r . & &
\end{array}
$$

\footnotetext{
${ }^{19}$ Note that in this case, as with $A d S$, the spectrum of fluctuations is known 31] so in principle one could also adopt an effective action approach as in section 3.2. Since our interest is only to demonstrate applicability of the CWZ formalism to a second example, we don't pursue this here.
} 
Now we look at small perturbations around this configuration, where the boundary of the droplet is deformed from $x_{2}=0$ into $x_{2}=\varepsilon\left(x_{1}\right)^{20}$. Note that this deformation is area preserving if $\int_{-\infty}^{\infty} d x_{1} \varepsilon\left(x_{1}\right)=0$. Making such a deformation takes us into a new solution with new functions $z\left(x_{1}, x_{2}, y\right), V_{i}\left(x_{1}, x_{2}, y\right)$. The corresponding variations can be found from (2.5), (2.6); to first order in $\varepsilon\left(x_{1}\right)$ they are given by:

$$
\begin{aligned}
\delta z\left(x_{1}, x_{2}, y\right) & =-\frac{y^{2}}{\pi} \int d x_{1}^{\prime} \frac{\varepsilon\left(x_{1}^{\prime}\right)}{\left[\left(x_{1}-x_{1}^{\prime}\right)^{2}+r^{2}\right]^{2}} \equiv-y^{2} I_{1}, \\
\delta V_{1}\left(x_{1}, x_{2}, y\right) & =-\frac{x_{2}}{\pi} \int d x_{1}^{\prime} \frac{\varepsilon\left(x_{1}^{\prime}\right)}{\left[\left(x_{1}-x_{1}^{\prime}\right)^{2}+r^{2}\right]^{2}}=-x_{2} I_{1}, \\
\delta V_{2}\left(x_{1}, x_{2}, y\right) & =\frac{1}{\pi} \int d x_{1}^{\prime} \frac{\left(x_{1}-x_{1}^{\prime}\right) \varepsilon\left(x_{1}^{\prime}\right)}{\left[\left(x_{1}-x_{1}^{\prime}\right)^{2}+r^{2}\right]^{2}} \equiv I_{2} .
\end{aligned}
$$

We see that these expressions have the form of convolutions in the $x_{1}$ variable. It thus makes sense to perform a Fourier transform w.r.t. $x_{1}$ (keeping the other two variables intact), which will turn these convolutions into products. Thus for a general function $f\left(x_{1}, x_{2}, y\right)$ we will have:

$$
f\left(x_{1}, x_{2}, y\right)=\int \frac{d p}{2 \pi} e^{-i p x_{1}} \tilde{f}\left(p, x_{2}, y\right), \quad \tilde{f}\left(p, x_{2}, y\right) \equiv \int d x_{1} e^{i p x_{1}} f\left(x_{1}, x_{2}, y\right) .
$$

The $\delta h$ can be expressed via $\delta z$ from (2.7). All these variations should be substituted into (6.2), and eventually into the integral (6.5). The dependence on $p$ can be removed from the integrand by rescaling $r|p| \rightarrow r$, with the remaining $x_{2}, y$ integral giving rise to a constant prefactor. A very similar computation is carried out for the 5 -form part of the current. These computations are detailed in Appendix B. The final result is that the symplectic form is of the form:

$$
\omega \propto \int \frac{d p}{2 \pi} \frac{i}{p} \tilde{\varepsilon}(p) \wedge \tilde{\varepsilon}(-p) .
$$

From this symplectic form we get the Poisson brackets $\left\{\tilde{\varepsilon}(p), \tilde{\varepsilon}\left(p^{\prime}\right)\right\}$ and, by the Dirac prescription, the commutators. Here too we find that the commutator of $\varepsilon\left(x_{1}\right)$ and $\varepsilon\left(x_{1}^{\prime}\right)$ constitute a $U(1)$ Kac-Moody algebra, and are thus of the same form as one expects based on the fermion analysis (2.39) (to see this, one has to apply (2.39) around a circular droplet of very large radius $R$. In this limit, we can identify the plane wave variable $x_{1}$ with the arc length measured along the boundary of the droplet, and $\varepsilon\left(x_{1}\right)$ with the perturbation of the droplet radius). The commutators which we find (see Appendix B) seem to be a factor 2 of what one expects from (2.39). We believe that this numerical mismatch is due to extra boundary terms which should be added to (6.5), as we remarked before. This issue is currently under investigation, and we hope to report on it in a future publication.

\footnotetext{
${ }^{20}$ As we are looking at small perturbations, this description is enough, and we do not consider situations where the droplet boundary winds such that it has a few $x_{2}$ values for a specific $x_{1}$, or where the topology of the boundary is changed and some disconnected droplets appear.
} 


\section{Conclusions and discussion}

In this paper we have considered the LLM family of solutions and set up a general framework for its quantization. In particular, using the CWZ method, we have performed the quantization in the case of the $A d S_{5} \times S^{5}$ background and demonstrated its consistency with a more direct effective action approach. This has permitted us to construct a quantum Hilbert space associated to the LLM solutions that precisely matches that of the corresponding fermion picture at large $N$. This result allows us to identify the spacetime $x_{1}, x_{2}$ plane with the Fermi liquid phase space, in agreement with the original LLM proposal. Moreover, we have derived general expressions for the symplectic form that can, in principle, be applied to more complicated droplets.

The outlook for future developments is as follows. First of all, one should explore the issue of boundary terms to be added to the general symplectic form (6.5) derived in Sec. 6. As discussed in Sec. 6, this should also be used to complete our quantization around the plane wave background ${ }^{21}$. It will also be of interest to generalize our techniques so that they may be easily applied to droplets with more complicated shapes $^{22}$ as well as nontrivial topologies ${ }^{23}$.

Another interesting course of work is the application of these methods to additional families of supergravity solutions that arise in various contexts. One example is the family of [32] with nonzero axion and dilaton, in which the solutions correspond to changing the fermions participating in the Quantum Hall Effect into fractional statistics particles. It would be interesting to reproduce this behavior from the gravity point of view. Another example is the family of $1 / 2$ BPS M2 brane solutions of 11-dimensional SUGRA described by LLM (see also subsequent work [33]).

Another important class of solutions to which one might apply these methods are the 1/4 BPS D1-D5 solutions with angular momentum constructed in [34]. As these are supersymmetric, we once again expect a quantization, at least to the order considered in our case, to have meaning even within the context of the full theory. The results would be very interesting [35] and could have implications in the context of D1-D5 black hole entropy as well as suggestions made by Mathur et al. regarding the relation of these solutions to the D1-D5 black hole [36].

Finally, it would also be interesting to consider going beyond the semiclassical

\footnotetext{
${ }^{21}$ One could also try to perform a higher-order expansion around the half-plane droplet. Such a computation could be useful to determine if a boundary term in the symplectic form is indeed missing. Note that the lowest-order plane wave computation of Sec. 6 is not suitable for such a check, since every lowest-order term in (6.2), (6.4) integrates separately to an expression of the same functional form.

${ }^{22}$ In this respect, see [20] for a discussion of how to quantize fermion droplets correspoding to multiple-valued $r(\phi)$.

${ }^{23}$ For instance, in the case of an annulus surrounding the origin we expect to get two identical $U(1)$ Kac-Moody algebras, one for each boundary, as the only coupling between the degrees of freedom on each boundary is through the total area conservation rule.
} 
approximation in supergravity and working at finite $N$. Of course, as soon as $N$ is taken away from infinity we have to deal with corrections from string theory. As a result, proceeding along this direction seems very difficult. It is possible, though, that the correspondence to fermions contains hints as to how one might proceed since the fermion picture essentially tells us what the correct quantization should look like. In fact, it seems to indicate that whatever $1 / N$ corrections arise must somehow conspire to yield a free theory in the appropriate description. It would be fascinating to understand this in detail.

\section{Note added in proof}

We have recently found what caused the factor 2 mismatch in section 6 . The reason is that the four-form potentials are singular if the axial gauge (2.11) is used. The singularity occurs at the $y=0$ plane, where the spheres $S^{3}$ and $\tilde{S}^{3}$, whose volume forms enter the potentials, shrink to zero. To compensate for this singularity, a boundary term located at $y=0$ should be added to the symplectic form. More details can be found in [37], where the general droplet case is also analyzed in full.

\section{Acknowledgments}

We would like to thank O. Aharony, J. de Boer, A. Dhar, J. Esole, N. Iizuka, O. Lunin, J. Maldacena, L. Motl, A. Naqvi, I. Papadimitriou, S. Raju, K. Schoutens, A. Shomer, K. Skenderis, M. Smedback, and especially S. Minwalla for useful discussion. We would also like to thank A. Dhar and S. Minwalla for collaboration at an early stage of this work. L.G., K.P., and J.M. would like to thank the Tata Institute of Fundamental Research for providing a stimulating research atmosphere during the beginning stages of this work. L.M. would like to thank the Weizmann Institute of Science for hospitality during the final period of this work. The work of L.G., J.M., and K.P. is supported by DOE grant DE-FG01-91ER40654. The work of J.M. is also supported in part by an NSF Graduate Research Fellowship. The work of L.M. and V.R. is supported by Stichting FOM.

\section{A. Evaluation of the CWZ currents around $A d S_{5} \times S^{5}$}

In this appendix, we provide details for the computations reported in Sec. 5. Thus we will compute the symplectic currents (4.11), (4.12) and the symplectic form (4.8) for fluctuations about the $A d S_{5} \times S^{5}$ background parameterized by (3.11), (3.10), (3.13). We proceed by brute force, computing the nonvanishing components of the various tensors appearing in (4.11), (4.12). 
Let $\alpha, \beta, \gamma(a, b, c)$ denote the three angles of the $S^{3}\left(\tilde{S}^{3}\right)$ in (3.3). The volume forms of the $S^{3}$ 's will be written in terms of these variables as

$$
d \Omega_{3}=\sin ^{2} \alpha \sin \beta d \alpha \wedge d \beta \wedge d \gamma, \quad d \tilde{\Omega}_{3}=\sin ^{2} a \sin b d a \wedge d b \wedge d c .
$$

The only nonzero components of $\delta\left[g^{m n} \sqrt{-g}\right]$ are given by

$$
\begin{aligned}
\delta\left[g^{t t} \sqrt{-g}\right] & =-\sum_{n \neq 0} 2 a_{n}(|n|+1) e^{i n(\tilde{\phi}+t)} \frac{\tanh ^{2} \rho}{\cosh ^{2} \rho} \sqrt{-\bar{g}} \mathcal{C}_{n}, \\
\delta\left[g^{t \rho} \sqrt{-g}\right] & =-\sum_{n \neq 0} \frac{2 i n a_{n}}{|n|}(|n|+1) e^{i n(\tilde{\phi}+t)} \frac{\tanh \rho}{\cosh ^{2} \rho} \sqrt{-\bar{g}} \mathcal{C}_{n}, \\
\delta\left[g^{\rho \rho} \sqrt{-g}\right] & =\sum_{n \neq 0} 2 a_{n}(|n|+1) e^{i n(\tilde{\phi}+t)} \frac{\sqrt{-\bar{g}} \mathcal{C}_{n}}{\cosh ^{2} \rho} \\
\delta\left[g^{\alpha \alpha} \sqrt{-g}\right] & =\sum_{n \neq 0} 2 a_{n}(|n|+1) e^{i n(\tilde{\phi}+t)} \frac{\sqrt{-\bar{g}} \mathcal{C}_{n}}{\sinh ^{2} \rho} \\
\delta\left[g^{\beta \beta} \sqrt{-g}\right] & =\sum_{n \neq 0} 2 a_{n}(|n|+1) e^{i n(\tilde{\phi}+t)} \frac{\sqrt{-\bar{g}} \mathcal{C}_{n}}{\sinh ^{2} \rho \sin ^{2} \alpha}, \\
\delta\left[g^{\gamma \gamma} \sqrt{-g}\right] & =\sum_{n \neq 0} 2 a_{n}(|n|+1) e^{i n(\tilde{\phi}+t)} \frac{\sqrt{-\bar{g}} \mathcal{C}_{n}}{\sinh ^{2} \rho \sin ^{2} \alpha \sin ^{2} \beta},
\end{aligned}
$$

where

$$
\sqrt{-\bar{g}}=\sinh ^{3} \rho \cosh \rho \sin ^{3} \theta \cos \theta \sin ^{2} \alpha \sin \beta \sin ^{2} a \sin b
$$

is the square root of the determinant of the unperturbed $A d S_{5} \times S^{5}$ metric and

$$
\mathcal{C}_{n} \equiv\left(\frac{\cos \theta}{\cosh \rho}\right)^{|n|}
$$

Using these, we find that the only nonzero $\delta \Gamma_{m n}^{t}$ which contribute to the first term 
of $J_{G}^{t}$ are:

$$
\begin{aligned}
\delta \Gamma_{t t}^{t} & =-\frac{1}{2} \sum_{n \neq 0} \frac{i n a_{n}}{|n|} e^{i n(\tilde{\phi}+t)}(|n|+1)\left(|n|+4-2 \frac{|n|+2}{\cosh ^{2} \rho}\right) \mathcal{C}_{n}, \\
\delta \Gamma_{t \rho}^{t} & =\frac{1}{2} \sum_{n \neq 0} a_{n} e^{i n(\tilde{\phi}+t)}(|n|+1)\left(|n|-2 \frac{|n|+2}{\cosh ^{2} \rho}\right) \tanh \rho \mathcal{C}_{n}, \\
\delta \Gamma_{\rho \rho}^{t} & =-\frac{1}{2} \sum_{n \neq 0} \frac{i n a_{n}}{|n|} e^{i n(\tilde{\phi}+t)}(|n|+1)\left(3|n|-2 \frac{|n|+2}{\cosh ^{2} \rho}\right) \frac{\mathcal{C}_{n}}{\cosh ^{2} \rho}, \\
\delta \Gamma_{\alpha \alpha}^{t} & =-\frac{1}{2} \sum_{n \neq 0} \frac{i n a_{n}}{|n|} e^{i n(\tilde{\phi}+t)}(|n|+1)(|n|-4) \tanh ^{2} \rho \mathcal{C}_{n}, \\
\delta \Gamma_{\beta \beta}^{t} & =-\frac{1}{2} \sum_{n \neq 0} \frac{i n a_{n}}{|n|} e^{i n(\tilde{\phi}+t)}(|n|+1)(|n|-4) \tanh ^{2} \rho \sin ^{2} \alpha \mathcal{C}_{n}, \\
\delta \Gamma_{\gamma \gamma}^{t} & =-\frac{1}{2} \sum_{n \neq 0} \frac{i n a_{n}}{|n|} e^{i n(\tilde{\phi}+t)}(|n|+1)(|n|-4) \tanh ^{2} \rho \sin ^{2} \alpha \sin ^{2} \beta \mathcal{C}_{n},
\end{aligned}
$$

while the only nonzero $\delta \Gamma_{m p}^{m}$ that contribute to the second term of $J_{G}^{t}$ are:

$$
\begin{aligned}
\delta \Gamma_{m t}^{m} & =\sum_{n \neq 0} i n a_{n} e^{i n(\tilde{\phi}+t)}(|n|+1) \mathcal{C}_{n}, \\
\delta \Gamma_{m \rho}^{m} & =-\sum_{n \neq 0} a_{n} e^{i n(\tilde{\phi}+t)}|n|(|n|+1) \tanh \rho \mathcal{C}_{n} .
\end{aligned}
$$

It is now a trivial matter to compute $J_{G}^{t}$. We find it easier to present $\int d \tilde{\phi} J_{G}^{t}$ rather than $J_{G}^{t}$ itself. From the first term, we get

$$
\begin{aligned}
& \int d \tilde{\phi} \delta \Gamma_{m p}^{t} \wedge \delta\left[\sqrt{-g} g^{m p}\right] \\
& =\sum_{n \neq 0} \frac{4 \pi i n(|n|+1)^{2} \mathcal{C}_{n}^{2}}{|n| \cosh ^{6} \rho}[7+|n|-3|n| \cosh (2 \rho)+\cosh (4 \rho)] \sqrt{-\bar{g}}\left(a_{n} \wedge a_{-n}\right)
\end{aligned}
$$

Performing the remaining integrals, we find

$$
\int_{t=\mathrm{const}} \delta \Gamma_{m p}^{t} \wedge \delta\left[\sqrt{-g} g^{m p}\right]=-\sum_{n \neq 0} \frac{2 \pi i\left(2 \pi^{2}\right)^{2}\left(n^{2}-|n|-8\right)}{(|n|-1)(|n|+2) n} a_{n} \wedge a_{-n}
$$

For the second term contributing to $J_{G}^{t}$, we have

$$
\int d \tilde{\phi} \delta \Gamma_{m p}^{p} \wedge \delta\left[\sqrt{-g} g^{m t}\right]=-\sum_{n \neq 0} \frac{8 \pi i n \mathcal{C}_{n}^{2} \tanh ^{2} \rho}{\cosh ^{2} \rho}(1+|n|)^{2} \sqrt{-\bar{g}}\left(a_{n} \wedge a_{-n}\right)
$$

Performing the remaining integrals here, we find

$$
\int_{t=\mathrm{const}} \delta \Gamma_{m p}^{p} \wedge \delta\left[\sqrt{-g} g^{m t}\right]=-\sum_{n \neq 0} \frac{4 \pi i\left(2 \pi^{2}\right)^{2}|n|}{(|n|-1)(|n|+2) n}\left(a_{n} \wedge a_{-n}\right)
$$


Combining (A.8) and (A.10), we obtain precisely Eq. (5.1) of Sec. 5 .

We now turn to the 5 -form current. From the expression (3.13) we can compute the perturbation of the 5 -form itself

$$
\begin{aligned}
& \delta F_{5}=\sum_{n \neq 0} a_{n} \mathcal{C}_{n} e^{i n(\tilde{\phi}+t)}\left(\frac{|n|+1}{2|n|}\right) \times \\
& \times\left[\left(|n|(|n|-4) \cosh \rho \sinh ^{3} \rho d t \wedge d \rho-n^{2} \sinh ^{2} \rho \tanh \rho d \rho \wedge d \tilde{\phi}+n^{2} \sinh ^{4} \rho \tan \theta d t \wedge d \theta\right.\right. \\
& \left.\quad-i n|n| \sinh ^{4} \rho d t \wedge d \tilde{\phi}-i n|n| \sinh ^{2} \rho \tanh \rho \tan \theta d \rho \wedge d \theta\right) \wedge d \Omega_{3} \\
& +\left(|n|(|n|+4) \cos \theta \sin ^{3} \theta d \theta \wedge d \tilde{\phi}-n^{2} \sin ^{2} \theta \tan \theta d t \wedge d \theta-n^{2} \sin ^{4} \theta \tanh \rho d \rho \wedge d \tilde{\phi}\right. \\
& \left.\left.\quad+i n|n| \sin ^{4} \theta d t \wedge d \tilde{\phi}-i n|n| \sin ^{2} \theta \tan \theta \tanh \rho d \rho \wedge d \theta\right) \wedge d \tilde{\Omega}_{3}\right] \quad(\text { A.11) }
\end{aligned}
$$

The variations that contribute to (4.12) (in this computation we haven't made use of the simplified form (4.14)) are as follows

$$
\begin{gathered}
\delta A_{\rho \alpha \beta \gamma}=-\sum_{n \neq 0} i n a_{n} \mathcal{C}_{n} e^{i n(\tilde{\phi}+t)}\left(\frac{|n|+1}{2|n|}\right) \sinh ^{2} \rho \tanh \rho \sin ^{2} \alpha \sin \beta \\
\delta A_{\tilde{\phi} a b c}=\sum_{n \neq 0}|n| a_{n} \mathcal{C}_{n} e^{i n(\tilde{\phi}+t)}\left(\frac{|n|+1}{2|n|}\right) \sin ^{4} \theta \sin ^{2} a \sin b \\
\delta A_{\theta a b c}=\sum_{n \neq 0} i n a_{n} \mathcal{C}_{n} e^{i n(\tilde{\phi}+t)}\left(\frac{|n|+1}{2|n|}\right) \sin ^{2} \theta \tan \theta \sin ^{2} a \sin b \\
\delta\left[\sqrt{-g} F^{t \rho \alpha \beta \gamma}\right]=-\sum_{n \neq 0} \frac{a_{n} \mathcal{C}_{n}}{2} e^{i n(\tilde{\phi}+t)}(|n|+1)(|n|+4) \cos \theta \sin ^{3} \theta \sin ^{2} a \sin b \\
\delta\left[\sqrt{-g} F^{t \tilde{\phi} a b c}\right]=-\sum_{n \neq 0} \frac{a_{n} \mathcal{C}_{n}}{2} e^{i n(\tilde{\phi}+t)} i n(|n|+1) \tanh \rho \sinh ^{2} \rho \sin ^{2} \alpha \sin \beta \tan \theta \\
\delta\left[\sqrt{-g} F^{t \theta a b c}\right]=\sum_{n \neq 0} \frac{a_{n} \mathcal{C}_{n}}{2} e^{i n(\tilde{\phi}+t)}|n|(|n|+1) \tanh \rho \sinh ^{2} \rho \sin ^{2} \alpha \sin \beta
\end{gathered}
$$

With this, we find that

$$
\int d \tilde{\phi} \delta A_{|\ldots|} \wedge \delta\left[\sqrt{-g} F^{t|\ldots|}\right]=\sum_{n \neq 0} \frac{i \pi n(1+|n|)^{2} \mathcal{C}_{n}^{2}[1+|n|+\cos (2 \theta)]}{|n| \cos ^{2} \theta \cosh ^{2} \rho} \sqrt{-\bar{g}}\left(a_{n} \wedge a_{-n}\right)
$$

Performing the remaining integrals, we reproduce Eq. (5.2) of Sec. 5. 


\section{B. Evaluation of the CWZ currents around the plane wave}

In this appendix we detail the derivation of the symplectic form around the plane wave background, outlined in Sec. 6. Let us start from the gravitational part of the symplectic form. We need expressions for all variations entering (6.2) in terms of $I_{1,2}$. Such expressions can be obtained using (2.7)-(2.8) and (6.7). We have:

$$
\begin{aligned}
\delta h^{-4} & =-16 r^{3} x_{2} I_{1}, \\
\delta\left(V_{i} z_{, i}\right) & =\frac{y^{2}}{2 r^{3}}\left(I_{2}+r^{2} \partial_{1} I_{1}\right), \\
\delta W_{12} & =\partial_{1} I_{2}+\partial_{2}\left(x_{2} I_{1}\right) .
\end{aligned}
$$

We use the Fourier transforms of the integrals $I_{1}, I_{2}$ given by:

$$
\begin{aligned}
& \tilde{I}_{1}\left(p, x_{2}, y\right)=\frac{1+r|p|}{2 r^{3}} e^{-r|p|} \tilde{\varepsilon}(p) \\
& \tilde{I}_{2}\left(p, x_{2}, y\right)=\frac{i p}{2 r} e^{-r|p|} \tilde{\varepsilon}(p)
\end{aligned}
$$

Then the first term in (6.2) gives the following contribution to the integral in (6.5):

$$
\begin{aligned}
& \int d x_{1} d x_{2} d y y^{3}\left[\frac{4 r^{2}\left(3 r^{2}+x_{2}^{2}\right)}{y^{4}}\right] \frac{y^{2}}{2 r^{3}}\left(I_{2}+r^{2} \partial_{1} I_{1}\right)\left(-y^{2} I_{1}\right) \\
& =\int \frac{d p}{2 \pi} \int d x_{2} d y\left(3 r^{2}+x_{2}^{2}\right) \frac{-2 y^{3}}{r}\left[\tilde{I}_{2}(p)+r^{2}(-i p) \tilde{I}_{1}(p)\right] \tilde{I}_{1}(-p) \\
& =\int \frac{d p}{2 \pi} \int d x_{2} d y\left(3+\frac{x_{2}^{2}}{r^{2}}\right) \frac{y^{3}}{2 r^{2}}(1+r|p|) e^{-2 r|p|} i p|p| \tilde{\varepsilon}(p) \tilde{\varepsilon}(-p) \\
& =\int d x_{2} d y\left(3+\frac{x_{2}^{2}}{r^{2}}\right) \frac{y^{3}}{2 r^{2}}(1+r) e^{-2 r} \times \Omega \equiv c_{1} \Omega, \\
& \Omega \equiv \int \frac{d p}{2 \pi} \frac{i}{p} \tilde{\varepsilon}(p) \tilde{\varepsilon}(-p),
\end{aligned}
$$

where we rescaled variables $\left(x_{2}, y\right) \rightarrow\left(x_{2}, y\right) /|p|$ to remove all dependence on $p$ from the $x_{2}, y$ integral, which became a constant prefactor. This prefactor can be easily evaluated:

$$
c_{1}=\int_{0}^{\pi} d \phi \int_{0}^{\infty} d r r\left(3+\cos ^{2} \phi\right) \frac{r \sin ^{3} \phi}{2}(1+r) e^{-2 r}=\frac{4}{3} .
$$

(the angular integral is from 0 to $\pi$ because of $y>0$ ).

Using that $V_{2}=0$ for the plane wave, the second term in (6.2) can be simplified as follows:

$$
\begin{aligned}
y^{3}\left[\delta\left(h^{-4} W_{12} V_{2}\right) \delta V_{1}-\delta\left(h^{-4} W_{12} V_{1}\right) \delta V_{2}\right] \\
=-y^{3}\left[2 W_{12} h^{-4} \delta V_{1} \delta V_{2}+W_{12} V_{1} \delta h^{-4} \delta V_{2}+h^{-4} V_{1} \delta W_{12} \delta V_{2}\right] .
\end{aligned}
$$


The first two terms here cancel because of $2 h^{-4} \delta V_{1}+V_{1} \delta h^{-4}=0$, as one can check using (6.6), (6.7), (B.1). The remaining term gives the following contribution to the integral in (6.5):

$$
\begin{aligned}
& \int d x_{1} d x_{2} d y 2 r y^{3}\left[\partial_{1} I_{2}+\partial_{2}\left(x_{2} I_{1}\right)\right] I_{2} \\
& =\int \frac{d p}{2 \pi} \int d x_{2} d y 2 r y^{3}\left[-i p \tilde{I}_{2}(p)+\partial_{2}\left(x_{2} \tilde{I}_{1}(p)\right)\right] \tilde{I}_{2}(-p) \\
& =\int \frac{d p}{2 \pi} \int d x_{2} d y \frac{-y^{3}}{2 r^{3}}\left[1+r|p|+r^{2} p^{2}-\frac{x_{2}^{2}}{r^{2}}\left(3+3 r|p|+r^{2} p^{2}\right)\right] e^{-2 r|p|} i p \tilde{\varepsilon}(p) \tilde{\varepsilon}(-p) \\
& \equiv c_{2} \Omega \\
& c_{2}=-\int d x_{2} d y \frac{y^{3}}{2 r^{3}}\left[1+r+r^{2}-\frac{x_{2}^{2}}{r^{2}}\left(3+3 r+r^{2}\right)\right] e^{-2 r}=-\frac{1}{3},
\end{aligned}
$$

where we have used the same method of computing the integral as in (B.3), (B.4).

Let us now proceed with the gauge field part of the symplectic current, (6.4). Expressions for the gauge fields were given in (2.11); we have to find their variations. Using (6.7) it is easy to show that

$$
\begin{aligned}
& \delta \frac{y^{2} V_{1}}{\frac{1}{2} \pm z}=2 r^{2} x_{2} I_{1} \mp 2 r^{3} I_{1}, \\
& \delta \frac{y^{2} V_{2}}{\frac{1}{2} \pm z}=2 r^{2} I_{2} \mp 2 r x_{2} I_{2} .
\end{aligned}
$$

We also have

$$
\begin{aligned}
& \delta \frac{1}{\pi} \frac{x_{2}}{x^{2}+y^{2}} * Z=-\frac{x_{2}}{\pi} \int d x_{1}^{\prime} \frac{\varepsilon\left(x_{1}^{\prime}\right)}{\left(x_{1}-x_{1}^{\prime}\right)^{2}+r^{2}} \equiv-x_{2} I_{3}, \\
& \delta \frac{1}{\pi} \frac{x_{1}}{x^{2}+y^{2}} * Z=-\frac{1}{\pi} \int d x_{1}^{\prime} \frac{\left(x_{1}-x_{1}^{\prime}\right) \varepsilon\left(x_{1}^{\prime}\right)}{\left(x_{1}-x_{1}^{\prime}\right)^{2}+r^{2}} \equiv-I_{4},
\end{aligned}
$$

with the corresponding Fourier transforms in $x_{1}$ given by

$$
\begin{aligned}
\tilde{I}_{3}\left(p, x_{2}, y\right) & =\frac{1}{r} e^{-r|p|} \tilde{\varepsilon}(p), \\
\tilde{I}_{4}\left(p, x_{2}, y\right) & =i \operatorname{sign} p e^{-r|p|} \tilde{\varepsilon}(p) .
\end{aligned}
$$

We thus have:

$$
\begin{aligned}
& -4 \delta B_{1}=\left(2 r^{2} x_{2} I_{1}-x_{2} I_{3}\right)+2 r^{3} I_{1} \equiv a_{1}+b_{1}, \\
& -4 \delta \tilde{B}_{1}=\left(2 r^{2} x_{2} I_{1}-x_{2} I_{3}\right)-2 r^{3} I_{1}=a_{1}-b_{1}, \\
& -4 \delta B_{2}=\left(2 r^{2} I_{2}+I_{4}\right)+2 r x_{2} I_{2} \equiv a_{2}+b_{2}, \\
& -4 \delta \tilde{B}_{2}=\left(2 r^{2} I_{2}+I_{4}\right)-2 r x_{2} I_{2}=a_{2}-b_{2} .
\end{aligned}
$$


The purpose of introducing this notation is that in (6.4) many cross-terms cancel:

$$
J_{F}^{t}=\left(\frac{\partial a_{2}}{\partial y} b_{1}-a_{2} \frac{\partial b_{1}}{\partial y}\right)-\left(\frac{\partial a_{1}}{\partial y} b_{2}-a_{1} \frac{\partial b_{2}}{\partial y}\right) .
$$

The Fourier transforms of $a_{i}, b_{i}$ are easily found using (B.2), (B.11); we have:

$$
\begin{aligned}
& \tilde{a}_{1}(p)=x_{2}|p| e^{-r|p|} \\
& \tilde{b}_{1}(p)=(1+|p| r) e^{-r|p|}, \\
& \tilde{a}_{2}(p)=i \operatorname{sign} p(1+|p| r) e^{-r|p|}=i \operatorname{sign} p \tilde{b}_{1}(p), \\
& \tilde{b}_{2}(p)=i x_{2} p e^{-r|p|}=i \operatorname{sign} p \tilde{a}_{1}(p) .
\end{aligned}
$$

Because of these proportionalities, substituting these expressions in $\int J_{F}^{t}$ results in a total cancellation (multiplication by $i \operatorname{sign} p$ will commute with the $y$ derivatives, and the integrands corresponding to both terms in the right-hand side of (B.13) will vanish on the Fourier side).

Thus we conclude that the gauge part does not contribute to the symplectic form around the plane wave (this is unlikely to be true in the general case). The final answer is given by adding the gravitational part contributions (B.4), (B.6); we have

$$
\omega=\frac{\left(2 \pi^{2}\right)^{2}}{2 \kappa_{10}^{2}} \Omega=\frac{2 \pi^{4}}{\kappa_{10}^{2}} \int \frac{d p}{2 \pi} \frac{i}{p} \tilde{\varepsilon}(p) \tilde{\varepsilon}(-p) .
$$

This symplectic form implies the commutators:

$$
\left[\tilde{\varepsilon}(p), \tilde{\varepsilon}\left(p^{\prime}\right)\right]=-\frac{\kappa_{10}^{2}}{2 \pi^{3}} p \delta\left(p+p^{\prime}\right) .
$$

From this it is easy to get the commutators in the coordinate representation:

$$
\left[\varepsilon\left(x_{1}\right), \varepsilon\left(x_{1}^{\prime}\right)\right]=-i \frac{\kappa_{10}^{2}}{4 \pi^{4}} \delta^{\prime}\left(x_{1}-x_{1}^{\prime}\right) .
$$

We would like to compare the last equation with the finite droplet result (2.39). For this we have to apply (2.39) around a circular droplet of very large radius $R$. In this limit, we can identify the plane wave variable $x_{1}$ with the arc length measured along the boundary of the droplet:

$$
x_{1}=-R \phi
$$

(the sign is chosen to preserve orientation) and $\varepsilon\left(x_{1}\right)$ with the perturbation in the droplet radius. Thus we have

$$
f^{2}(\phi) \approx R^{2}+2 R \varepsilon\left(x_{1}\right) .
$$

Using $\delta^{\prime}\left(\phi-\phi^{\prime}\right)=-R^{2} \delta^{\prime}\left(x_{1}-x_{1}^{\prime}\right)$, we see that (2.39) in the limit $R \rightarrow \infty$ gives the result which is of precisely the same functional form as (B.17), but contains an extra factor $1 / 2$ in the right-hand side. We suspect that the reason for this mismatch, as we mentioned in Sec. 6, is that there should be a boundary term added to the symplectic form, which would give an equal contribution as the bulk term we have just evaluated. 


\section{References}

[1] J. M. Maldacena, "The large N limit of superconformal field theories and supergravity," Adv. Theor. Math. Phys. 2, 231 (1998) [Int. J. Theor. Phys. 38, 1113 (1999)] [arXiv:hep-th/9711200].

S. S. Gubser, I. R. Klebanov and A. M. Polyakov, "Gauge theory correlators from non-critical string theory," Phys. Lett. B 428, 105 (1998) [arXiv:hep-th/9802109]. E. Witten, "Anti-de Sitter space and holography," Adv. Theor. Math. Phys. 2, 253 (1998) [arXiv:hep-th/9802150].

[2] S. Corley, A. Jevicki and S. Ramgoolam, "Exact correlators of giant gravitons from dual N = 4 SYM theory," Adv. Theor. Math. Phys. 5, 809 (2002) [arXiv:hep-th/0111222].

[3] D. Berenstein, "A toy model for the AdS/CFT correspondence," JHEP 0407, 018 (2004) [arXiv:hep-th/0403110].

[4] H. Lin, O. Lunin and J. Maldacena, "Bubbling AdS space and 1/2 BPS geometries," JHEP 0410, 025 (2004) [arXiv:hep-th/0409174].

[5] G. Mandal, "Fermions from half-BPS supergravity," arXiv:hep-th/0502104.

[6] H. J. Kim, L. J. Romans and P. van Nieuwenhuizen, "The Mass Spectrum Of Chiral $\mathcal{N}=2 D=10$ Supergravity On $S^{5}$," Phys. Rev. D 32, 389 (1985).

[7] S. M. Lee, S. Minwalla, M. Rangamani and N. Seiberg, "Three-point functions of chiral operators in $D=4, \mathcal{N}=4$ SYM at large $N$," Adv. Theor. Math. Phys. 2, 697 (1998) [arXiv:hep-th/9806074].

[8] R. Arnowitt, S. Deser and C. W. Misner, "The Dynamics Of General Relativity," in Gravitation: an introduction to current research, Ed. L. Witten (Wiley 1962), p.227 [arXiv:gr-qc/0405109].

[9] Č. Crnković and E. Witten, "Covariant Description Of Canonical Formalism In Geometrical Theories," in Three hundred years of gravitation, Eds. S.W. Hawking and W. Israel (Cambridge University Press, 1987), p.676.

[10] G. J. Zuckerman, "Action Principles And Global Geometry," in Mathematical Aspects Of String Theory, San Diego 1986, Proceedings, Ed. S.-T. Yau (Worls Scientific, 1987), p.259.

[11] C. W. Misner, "Minisuperspace," in Magic Without Magic: John Archibald Wheeler, Ed. J. R. Klauder (Freeman, San Francisco 1972), p. 441.

[12] J. A. Wheeler, "Geometrodynamics and the issue of the final state," in Relativity, Groups and Topology, Eds. C. DeWitt and B. DeWitt (Gordon and Breach, New York, 1964) 
[13] B. S. DeWitt, "Quantum Theory Of Gravity. I. The Canonical Theory," Phys. Rev. 160, 1113 (1967).

[14] K. Kuchař, "Canonical Quantization Of Cylindrical Gravitational Waves," Phys. Rev. D 4, 955 (1971). G. A. Mena Marugán and M. Montejo, "Quantization of pure gravitational plane waves," Phys. Rev. D 58, 104017 (1998) [arXiv:gr-qc/9806105].

[15] J. Kinney, J. Maldacena and S. Minwalla, to appear.

[16] I. R. Klebanov, "TASI lectures: Introduction to the AdS/CFT correspondence," arXiv:hep-th/0009139.

[17] N. Itzhaki and J. McGreevy, "The large $N$ harmonic oscillator as a string theory," Phys. Rev. D 71, 025003 (2005) [arXiv:hep-th/0408180].

[18] D. Berenstein, "A matrix model for a quantum Hall droplet with manifest particle-hole symmetry,", Phys. Rev. D 71 (2005) 085001 [arXiv:hep-th/0409115].

[19] X.-G. Wen, "Topological orders and Edge excitations in FQH states", arXiv:cond-mat/9506066.

[20] A. Dhar, "Bosonization of non-relativstic fermions in 2-dimensions and collective field theory," arXiv:hep-th/0505084.

[21] A. P. Polychronakos, "Chiral actions from phase space (quantum Hall) droplets," Nucl. Phys. B 705, 457 (2005) [arXiv:hep-th/0408194].

[22] F. D. M. Haldane, "Stability of Chiral Luttinger Liquids and Abelian Quantum Hall States," Phys. Rev. Lett. 74, 2090 (1995) [arXiv:cond-mat/9501007].

[23] G. Dall'Agata, K. Lechner and D. P. Sorokin, "Covariant actions for the bosonic sector of D = 10 IIB supergravity," Class. Quant. Grav. 14 (1997) L195 [arXiv:hep-th/9707044].

[24] E. Witten, "Interacting Field Theory Of Open Superstrings," Nucl. Phys. B 276, 291 (1986).

[25] M. f. Chu, P. Goddard, I. Halliday, D. I. Olive and A. Schwimmer, "Quantization of the Wess-Zumino-Witten model on a circle," Phys. Lett. B 266, 71 (1991).

Y. Nutku, "Lagrangian approach to integrable systems yields new symplectic structure for KdV," in Integrable hierarchies and modern physical theories, Chicago 2000, Proceedings, Eds. H. Aratyn and A. Sorin (Kluwer, 2001), p.203 [arXiv:hep-th/0011052].

J. Lucietti, "Canonical quantization of a massive particle on AdS(3)," JHEP 0305 (2003) 017 [arXiv:hep-th/0303228].

[26] J. Lee and R. M. Wald, "Local Symmetries And Constraints," J. Math. Phys. 31, 725 (1990).

B. Julia and S. Silva, "On covariant phase space methods," arXiv:hep-th/0205072. 
[27] G. W. Gibbons and S. W. Hawking, "Action Integrals And Partition Functions In Quantum Gravity," Phys. Rev. D 15, 2752 (1977).

[28] L. D. Landau and E. M. Lifshitz, The Classical Theory of Fields, (Butterworth-Heinemann, 1980).

[29] J. L. Friedman, "Generic Instability of Rotating Relativistic Stars," Comm. Math. Phys. 62, 247 (1978).

[30] R. M. Wald and A. Zoupas, "A General Definition of "Conserved Quantities" in General Relativity and Other Theories of Gravity," Phys. Rev. D 61, 084027 (2000) [arXiv:gr-qc/9911095].

[31] R. R. Metsaev and A. A. Tseytlin, "Exactly solvable model of superstring in plane wave Ramond-Ramond background," Phys. Rev. D 65, 126004 (2002) [arXiv:hep-th/0202109].

[32] J. T. Liu, D. Vaman and W. Y. Wen, "Bubbling 1/4 BPS solutions in type IIB and supergravity reductions on $S^{n} \times S^{n}$," arXiv:hep-th/0412043.

J. T. Liu and D. Vaman, "Bubbling 1/2 BPS solutions of minimal six-dimensional supergravity," arXiv:hep-th/0412242.

[33] D. Bak, S. Siwach and H. U. Yee, "1/2 BPS geometries of M2 giant gravitons," arXiv:hep-th/0504098.

[34] O. Lunin, J. Maldacena and L. Maoz, "Gravity solutions for the D1-D5 system with angular momentum," arXiv:hep-th/0212210.

[35] L. Maoz, V. S. Rychkov and A. Shomer, in progress.

[36] S. D. Mathur "The fuzzball proposal for black holes: An elementary review," arXiv:hep-th/0502050.

O. Lunin and S. D. Mathur, "AdS/CFT duality and the black hole information paradox," Nucl. Phys. B 623, 342 (2002) [arXiv:hep-th/0109154].

[37] L. Maoz and V. S. Rychkov, "Geometry quantization from supergravity: The case of 'Bubbling AdS'," arXiv:hep-th/0508059. 